\title{
Normalwerte der Porphyrine und Porphyrin-Vorstufen
}

\author{
Von L. GoreczKy, I. Róth und M. BreckNer \\ Aus dem Zentrallaboratorium und der III. Medizinischen Abteilung des MAV-Krankenbauses, Budapest, Ungarn
}

(Eingegangen am 29. Juni 1968)

\begin{abstract}
Die Autoren bestimmten mit den bei Porphyrinopathien angewandten Methoden die Normalwerte von Proto-, Uro- und Koproporphyrin sowie inrer Vorstufen in Erythrocyten, Plasma, Galle, Harn und Faeces von 20-50 gesunden Personen und verglichen sie mit den in der Literatur angegebenen Werten.
\end{abstract}

\section{Normal values for the concentrations of porpbyrins and porphyrin precursors}

With the methods routinely employed for porphyrinopathias, normal values were measured on $20-50$ healthy persons and compared with the literature values.

Die Differenzierung der hepatischen und erythropoetischen Formen der Porphyrinopathien rückt immer mehr in den Vordergrund und damit immer mehr auch die Bedeutung der Abgrenzung der einzelnen Krankheitsformen. Die Klärung der einzelnen Krankheitsbilder erfordert Untersuchungen, die sich in die Laboratoriums-Routinearbeit schwer einfügen lassen. Die Forschungen der Biochemie begründeten weitgehend die Bestimmung der Porphyrine und ihrer Vorstufen. Die diesbezüglichen Untersuchungsmethoden erfuhren in den letzten zehn Jahren eine bedeutende Entwicklung. Die neuen Verfahren erleichtern infolge ihrer Empfindlichkeit und Genauigkeit in hohem Maße die Trennung der Krankheitsbilder und ermöglichen zugleich die Bestimmung auch ganz geringer Porphyrinmengen, weshalb für die Untersuchung bedeutend weniger Material erforderlich ist. Die Fortentwicklung der Methoden brachte naturgemäß auch Änderungen hinsichtlich der Normalwerte mit sich. Dies zeigt sich in den Resultaten von ZIEVE und Mitarbeitern (1), die mit Hilfe ihres verbesserten neuen Verfahrens im Harn einen Koproporphyrinwert von $54 \mu \mathrm{g}$ pro die, gegenüber einem von $160 \mu \mathrm{g}$ pro die feststellen konnten. Das gleiche gilt für die früher und die später im Stuhl gefundenen. Werte von EALES $(2,3)$; bei den früheren Bestimmungen sind Werte von $7,6 \mu \mathrm{g} / \mathrm{g}$, bzw. $29 \mu \mathrm{g} / \mathrm{g}$ für Kopro-, bzw. Protoporphyrin angegeben, in späteren aber hat der genannte Autor 0-110 bzw. $0-37 \mu \mathrm{g} / \mathrm{g}$ Faeces-Trockenrückstand gefunden.

Die Normalwerte weichen je nach Bestimmungsmethode voneinander $a b$ und lassen sich daher den Angaben der ausländischen Literatur nicht mit voller Gültigkeit gegenüberstellen. Das beste Beispiel hierfür liefert eine kürzlich erschienene Mitteilung aus Südafrika (4), in welcher die Gesamtmenge des Porphyrins im Stuhl mit $200 \mu \mathrm{g} / \mathrm{g}$ Trockenrückstand angegeben wird. Dagegen liegt eine Mitteilung aus Schweden von HAEGER-Aronsèn (5) vor, wonach der Mittelwert des Koporporphyrins $2 \mu \mathrm{g} / \mathrm{g}$ Trockenrückstand und der Höchstwert $6 \mu \mathrm{g} / \mathrm{g}$ beträgt. Wir selbst konnten in eigenen Untersuchungen für Ungarn einen höheren Mittelwert feststellen. Auch bestehen Unterschiede in den Bezugsgrößen für die einzelnen Werte, wobei den Werten mancher Mitteilungen die entleerte Menge pro
Tag, denen anderer jedoch pro Liter zugrunde liegt. Für die Stuhlporphyrine findet man die Werte pro die, pro $100 \mathrm{~g}$ bzw. auf Trockengewicht bezogen ausgedrückt. Einheitlichkeitshalber jedoch ist ohne Zweifel die Angabe pro $g$ Trockengewicht angemessener.

Unzulänglichkeiten beim Sammeln und Lagern der Exkremente für die Porphyrinbestimmung können mit hohem Materialverlust einhergehen, der nach HiNsBerg und LANG (6) in Urin mitunter $40-50 \%$ erreichen kann. Auch machten wir die Erfahrung, daß die Vorschriften für das Sammeln des Untersuchungsmaterials von den klinischen Abteilungen nicht mit der erforderlichen Sorgfalt eingehalten werden, obgleich - wie auch BRUGSCH (7) feststellt - Möglichkeiten bestehen, das Material zu konservieren. Wie auch andere Autoren fordern, ist es in diesen Fällen am zweckmäßigsten die Bestimmungen in frischem Morgenurin durchzuführen und die Werte pro Liter anzugeben.

Bakterielle Infektionen können gleichfalls zu hohen Verlusten führen. Ferner sei betont, daß der Stuhl unverzüglich aufzuarbeiten ist, da es sonst zur Bildung verschiedener Abbauprodukte des Porphyrins kommen kann, wodurch die Ergebnisse weitgehend verfälscht werden können. Um dies zu vermeiden, lagern einige Autoren den Stuhl bis zur Aufarbeitung bei -20 $0^{\circ}$ (8).

Bei der Bestimmung in roten Blutkörperchen wird der Porphyrinwert von manchen Autoren auf $100 \mathrm{ml}$ Erythrocytenkonzentrat, von anderen auf ein rotes Blutkörperchen bezogen. Die Werte sind dementsprechend sehr unterschiedlich, lassen sich aber bei Kenntnis des Hämatokritwertes und der Zahl der roten Blutkörperchen entsprechend umrechnen.

Die Mitteilungen mancher Autoren enthalten zuweilen auch die mit ihrem Verfahren bestimmten Normalwerte und ermöglichen damit die richtige Beurteilung ihrer Ergebnisse; andere Autoren wieder legen Normalwerte anderer Untersucher zugrunde.

Die Normalwerte bei Säuglingen und Kindern sind anders zu bewerten, da sie bedeutend niedriger sind als bei Erwachsenen (KäsER und Mitarbeiter (9); Politzer und Kessel (10)).

Aufgrund unserer seit 20 Jahren durchgeführten Untersuchungen konnten wir die in der Literatur mitgeteilten Angaben mit denjenigen für Ungarn ver- 
gleichen. Dies war notwendig, weil sich die Angaben der Normalwerte für die einzelnen Porphyrine und Exkremente je nach Land und Untersucher in ziemlich breiten Grenzen bewegen, was zum Teil auf rassische, zum Teil auf Ernährungsfaktoren zurückgeführt wird (4). Erwähnenswerte Beweise liegen über den Zusammenhang zwischen Porphyrinausscheidung und Ernährung von BRUGSCH (7) vor.

Diese Tatsachen erschweren in jeder Hinsicht den Vergleich der Resultate einzelner Autoren der verschiedenen Länder hinsichtlich der Normalwerte. Deshalb fanden wir es notwendig unsere eigenen Normalwerte entsprechend aufzuarbeiten.

Im folgenden sind unsere mit den in der Diagnostik des Porphyrinopathien üblichen Methoden erhaltenen Ergebnisse angeführt, ebenso die angewandten Verfahren und Normalwerte, die an gesunden Personen bestimmt worden sind. Vergleichshalber sind die von anderen. Autoren angegebenen Normalwerte angeführt. Von der Besprechung qualitativer Proben sehen wir hier $a b$, da diese die quantitative Bestimmung nicht berühren.

\section{Normalwerte der Porphyrinvorstufen}

Bei der quantitativen Untersuchung der im Harn nachweisbaren Präkursoren wandten wir die Ionenaustausch-Methode nach MAUZERALI und Granick (11) an; bei der Untersuchung des Serums gingen wir nach dem Verfahren von Gibson und Mitarbeitern vor (12). Die Ergebnisse zeigen die Tabelle 1 und 2.

Tab. 1

Normalwerte von $\delta$-Aminolaevulinsäure in Harn, Serum und Erythrocyten. Die eigenen Ergebnisse werden mit in der Literatur angegebenen verglichen

\begin{tabular}{|c|c|c|c|}
\hline Material & Autor & $\begin{array}{l}\text { Zitat } \\
\text { Nr. }\end{array}$ & gefundene Menge \\
\hline \multirow[t]{2}{*}{ Urin } & $\begin{array}{l}\text { HAEGER-ARONSEN } \\
\text { HEILMEYER } \\
\text { MALOOLY u. Mitarbeiter } \\
\text { PETERKA u. Mitarbeiter } \\
\text { PETERS } \\
\text { PRATO u. Mitarbeiter } \\
\text { RENNER } \\
\text { STICH }\end{array}$ & $\begin{array}{r}5 \\
13 \\
14 \\
15 \\
16 \\
17 \\
18 \\
19\end{array}$ & $\begin{array}{ll}3050 \pm 130 & \mu \mathrm{g} / \mathrm{Tag} \\
2217 \pm 267 & \mu \mathrm{g} / \mathrm{Tag} \\
2630 \pm 135 & \mu \mathrm{g} / \mathrm{Tag} \\
400 & \mu \mathrm{g} / \mathrm{Tag} \\
2500 & \mu \mathrm{g} / \mathrm{Tag} \\
2844 \pm 1228 & \mu \mathrm{g} / \mathrm{Tag} \\
2130 \pm 415 & \mu \mathrm{g} / \mathrm{Tag} \\
2130 \pm 400 & \mu \mathrm{g} / \mathrm{Tag}\end{array}$ \\
\hline & RÓTH U. GORECZKY & 20 & $2220 \pm 340 \mu \mathrm{g} / \mathrm{Tag}$ \\
\hline \multirow[t]{2}{*}{ Serum } & HAEGER-ARONSEN & 21 & $19 \pm 4 \mu \mathrm{g} / 100 \mathrm{ml}$ \\
\hline & $\begin{array}{c}\text { GORECZKY, RóTh } \\
\text { BRECKNER }\end{array}$ & & $20 \pm 5 \mu \mathrm{g} / 100 \mathrm{ml}$ \\
\hline
\end{tabular}

Erythro-

cyten

HEILMEYER $\quad 13 \quad 30,9 \pm 10,8 \mu \mathrm{g} / 100 \mathrm{ml}$

Tab. 2

Normalwerte von Porphobilinogen im Harn. Die eigenen Ergebnisse werden mit in der Literatur angegebenen verglichen

\begin{tabular}{lcr}
\hline \multicolumn{1}{c}{ Autor } & Zitat Nr. & gefundene Menge \\
\hline HEILMEYER & 13 & $1513 \pm 220 \mu \mathrm{g} / \mathrm{Tag}$ \\
MALOLY u. Mitarbeiter & 14 & $1400 \pm 510 \mu \mathrm{g} / \mathrm{Tag}$ \\
PETERKA u. Mitarbeiter & 15 & 400 \\
PRATO u. Mitarbeiter & 17 & $671 \pm 414 \mu \mathrm{g} / \mathrm{Tag}$ \\
RENNER & 18 & $1550 \pm 170 \mu \mathrm{gg} / \mathrm{Tag}$ \\
STICH & 19 & $1550 \pm 170 \mu \mathrm{g} / \mathrm{Tag}$ \\
\hline RótH u. GORECZKY & 20 & $1550 \pm 170 \mu \mathrm{g} / \mathrm{Tag}$ \\
\hline
\end{tabular}

Normalwerte der Porphyrine

Die Bestimmung der Porphyrine geschieht spektrophotometrisch, die Grundlage dafür bietet ihre Löslichkeit. Nach der Isolierung werden die Porphyrine in Salzsäurelösungen nach der von RrMnNGTON und Mitarbeitern $(22,23)$, sowie von WrTH $(24)$ angegebenen
Vorschrift in der Soret-Bande bestimmt, da die Absorption hier maximal ist. Die Uroporphyrine wurden nach dem Verfahren von Rimington (25) bestimmt. Bezüglich der Ausscheidung sind die Angaben je nach Autor äußerst unterschiedlich (s. die Feststellungen von BrugsCH (7)). Im folgenden bringen wir die Normalwerte der einzelnen Porphyrine nach dem Untersuchungsmaterial gruppiert (Tab. 3-7).

Tab. 3

Normalwerte für Uroporphyrin, Koproporphyrin und Protoporphyrin im Harn. Die eigenen Ergebnisse werden mit in der Literatur angegebenen verglichen

\begin{tabular}{|c|c|c|c|c|}
\hline Porphyrin & Autor & $\begin{array}{l}\text { Zitat } \\
\text { Nr. }\end{array}$ & gefundene & Mengen \\
\hline \multirow[t]{4}{*}{ Uro- } & \multirow{3}{*}{$\begin{array}{l}\text { BRUGSCH } \\
\text { HEILMEYER } \\
\text { PETERKA u. Mitarbeiter } \\
\text { REINKINGH u. vAN } \\
\text { KAMPEN } \\
\text { WALDENSTRÖM u. } \\
\text { HAEGER-ARONSEN } \\
\text { WATSON u. Mitarbeiter }\end{array}$} & $\begin{array}{r}7 \\
13 \\
15\end{array}$ & \multicolumn{2}{|c|}{$\begin{array}{c}\text { nur in Spuren } \\
6,24 \pm 5,22 \mu \mathrm{g} / \mathrm{Tag} \\
5-30 \quad \mu \mathrm{g} / \mathrm{Tag}\end{array}$} \\
\hline & & 26 & $12,0 \pm 5$ & $\mu \mathrm{g} / \mathrm{Tag}$ \\
\hline & & $\begin{array}{l}27 \\
28\end{array}$ & $\begin{array}{l}110 \\
30\end{array}$ & $\underset{\mu \mathrm{g} / \mathrm{Tag}}{\mu \mathrm{Tag}}$ \\
\hline & \multicolumn{2}{|l|}{$\begin{array}{l}\text { GORECZKY, RóTH u. } \\
\text { BRECKNER }\end{array}$} & $1-25$ & $\mu \mathrm{g} / \mathrm{Tag}$ \\
\hline \multirow[t]{4}{*}{ Kopro- } & \multirow{3}{*}{$\begin{array}{l}\text { BRUGSCH } \\
\text { HEILMEYER } \\
\text { PETERKA u. Mitarbeiter } \\
\text { PRATO u. Mitarbeiter } \\
\text { REINKINGH u. vAN } \\
\text { KAMPEN } \\
\text { WALDENSTRÖM u. } \\
\text { HAEGER-ARONSEN } \\
\text { WATSON U. Mitarbeiter }\end{array}$} & $\begin{array}{r}7 \\
13 \\
15 \\
17\end{array}$ & \multicolumn{2}{|c|}{$\begin{array}{c}100 \\
72,6 \pm 31,3 \mu \mathrm{g} / \mathrm{Tag} / \mathrm{Tag} \\
100-250 \mu \mu \mathrm{g} / \mathrm{Tag} \\
102,4 \pm 29,2 \mu \mathrm{g} / \mathrm{Tag}\end{array}$} \\
\hline & & 26 & $36 \pm 15$ & $\mu \mathrm{g} / \mathrm{Tag}$ \\
\hline & & $\begin{array}{l}27 \\
28\end{array}$ & $\begin{array}{l}150 \\
275\end{array}$ & $\underset{\mu \mathrm{g} / \mathrm{Tag}}{\mu \mathrm{Tag}}$ \\
\hline & RóTH u. GORECZKY & 20 & $150 \pm 45$ & $\mu \mathrm{g} / \mathrm{Tag}$ \\
\hline Proto- & HEILMEYER u. ClotTEN & 29 & \multicolumn{2}{|c|}{ in Spuren } \\
\hline
\end{tabular}

Bei den angeführten Werten sind die Isomere nicht berücksichtigt worden; bekanntlich nämlich können das Isomere $I$ in minimalen Mengen vorkommen und auch 7, 6, 5 und 3 Carboxylgruppen enthaltende Porphyrine erscheinen, doch haben diese vor allem in pathologischen Fällen Bedeutung. Über die chromatographische und mit Sephadex durchführbare Trennung der Isomere und der ungerade Carboxylgruppenzahl enthaltenen Porphyrine liegt ein zusammenfassender Bericht von Schwartze (30) vor.

Tab. 4

Normalwerte für Uroporphyrin, Koproporphyrin und Protoporphyrin im Stuhl. Die eigenen Ergebnisse werden mit in der Literatur angegebenen verglichen

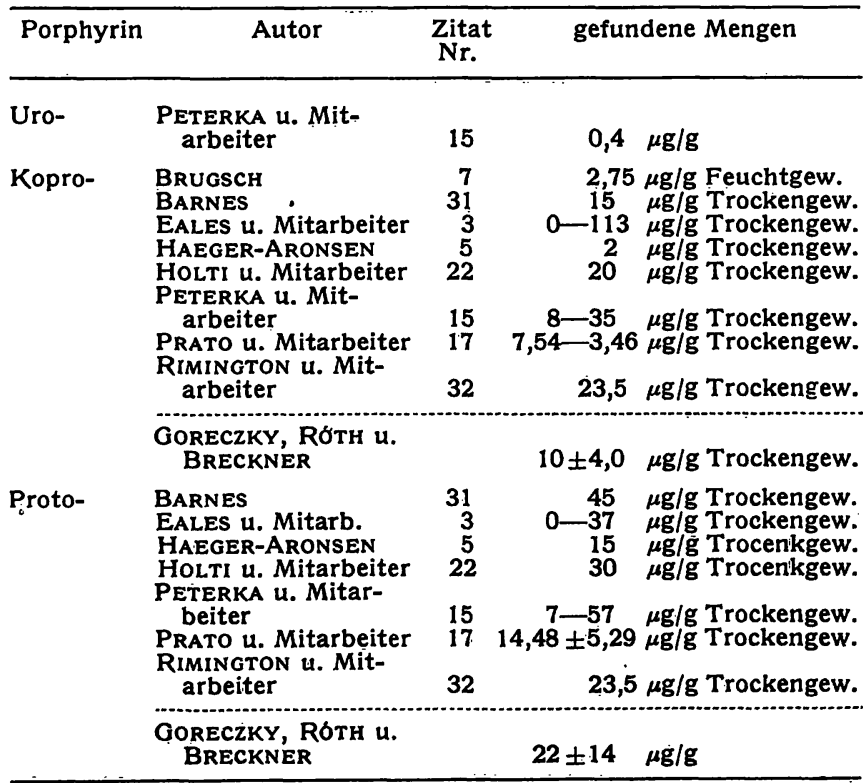




\section{Stubl}

Die stärksten Abweichungen zeigen sich in den Werten der im Stuhl vorkommenden Prophyrine; dieser Tatsache dürften gleichfalls die bereits erwähnten, von BrugsCH (7) festgestellten Ursachen zugrunde liegen.

Wir führten unsere Bestimmungen nach der Methode von HoLrr und Mitarbeitern (22) durch. $\mathrm{Da}_{\mathrm{a}}$ aber die im Stuhl anwesenden Farbstoffe das Ablesen zuweilen erschweren, haben wir das Verfahren modifiziert, indem wir nach der ersten Åther-Extraktion nach vorheriger Oxydation des Extraktes mit Jodlösung und Waschen mit Wasser - eine Extraktion mit 15proz. Salzsäure einfügten. Die Salzsäure-Extrakte wurden dann mit festem Natriumacetat auf pH 4,0 eingestellt (Kongopapier-Kontrolle) und die Porphyrine danach wieder in Ather ausgeschüttelt. Auf diese Weise gelang es, einen bedeutenden Teil der Verunreinigungen zu entfernen und den ersten Åtherauszug ohne Verlust zu reinigen. Aus dem zweiten Âtherextrakt wurden dann die einzelnen Porphyrinarten nach dem ursprünglichen Verfahren extrahiert.

\section{Erjithrocyten}

Die Bestimmung des Porphyringehaltes der roten Blutkörperchen geschah nach der von Rimingron und Mitarbeitern (32) angegebenen Methode. Einige Autoren schlagen vor, den Porphyrinwert für den einzelnen Erythrocyten anzugeben (33), allgemein jedoch findet man ihn in der Literatur auf $100 \mathrm{~m} l$ Erythrocytenkonzentrat bezogen.

Tab. 5

Normalwerte für Uroporphyrin, Koproporphyrin und Protoporphyrin in Erythrocyten. Die eigenen Ergebnisse werden mit in der Literatur angegebenen verglichen

\begin{tabular}{|c|c|c|c|c|}
\hline \multirow{3}{*}{$\begin{array}{l}\text { Porphyrin } \\
\text { Uro- } \\
\text { Kopro- }\end{array}$} & \multirow{2}{*}{ 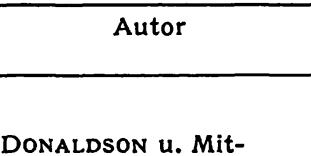 } & \multirow{2}{*}{$\begin{array}{l}\text { Zitat } \\
\text { Nr. }\end{array}$} & \multicolumn{2}{|c|}{ gefundene Mengen } \\
\hline & & & \multicolumn{2}{|c|}{ nur in Spuren } \\
\hline & $\begin{array}{l}\text { DONALDSON u. Mit- } \\
\text { arbeiter } \\
\text { KAUTZSCH u. MEYER } \\
\text { LANGHOF u. Mitarbeiter } \\
\text { PETERKA u. Mitarbeiter } \\
\text { HEILMEYER } \\
\text { PRATO u. Mitarbeiter } \\
\text { RIMINGTON u. Mit- } \\
\text { arbeiter }\end{array}$ & $\begin{array}{l}34 \\
33 \\
35 \\
15 \\
13 \\
17 \\
32\end{array}$ & $\begin{array}{c}0,5-2,3 \\
0,0-5,0 \\
0,0-5,0 \\
0,5-2,0 \\
1,62 \pm 2,38 \\
1,25 \pm 1,00 \\
0,35\end{array}$ & $\begin{array}{l}\mu \mathrm{g} / 100 \mathrm{ml} \\
\mu \mathrm{g} / 100 \mathrm{ml} \\
\mu \mathrm{g} / 100 \mathrm{ml} \\
\mu \mathrm{g} / 100 \mathrm{ml} \\
\mu \mathrm{g} / 100 \mathrm{ml} \\
\mu \mathrm{g} / 100 \mathrm{ml} \\
\mu \mathrm{g} / 100 \mathrm{ml}\end{array}$ \\
\hline & $\begin{array}{l}\text { GORECZKY, RÓTH u. } \\
\text { BRECKNER }\end{array}$ & & $2,89 \pm 1,51$ & $\mu \mathrm{g} / 100 \mathrm{ml}$ \\
\hline Proto- & $\begin{array}{l}\text { DONALDSON u. Mit- } \\
\text { arbeiter } \\
\text { DAGG u. Mitarbeiter } \\
\text { HEILMEYER } \\
\text { KAUTZSCH u. MEYER } \\
\text { LANGHOF u. Mitarbeiter } \\
\text { PETERKA u. Mitarbeiter } \\
\text { PRATO u. Mitarbeiter } \\
\text { RIMINGTON u. Mit- } \\
\text { arbeiter }\end{array}$ & $\begin{array}{l}34 \\
36 \\
13 \\
33 \\
35 \\
15 \\
17\end{array}$ & $\begin{array}{c}15-52 \\
15,5 \pm 5,7 \\
34,5 \pm 19,6 \\
15-35 \\
25-40 \\
15-65 \\
36,22 \pm 15,36 \\
34,5 \pm 19,6\end{array}$ & $\begin{array}{l}\mu \mathrm{g} / 100 \mathrm{ml} \\
\mu \mathrm{g} / 100 \mathrm{ml} \\
\mu \mathrm{g} / 100 \mathrm{ml} \\
\mu \mathrm{g} / 100 \mathrm{ml} \\
\mu \mathrm{g} / 100 \mathrm{ml} \\
\mu \mathrm{g} / 188 \mathrm{ml} \\
\mu \mathrm{g} / 100 \mathrm{ml} \\
\mu \mathrm{g} / 100 \mathrm{ml}\end{array}$ \\
\hline & $\begin{array}{l}\text { GORECZKY, RóTH u. } \\
\text { BRECKNER }\end{array}$ & & $22,5 \pm 10,6$ & $\mu \mathrm{g} / 100 \mathrm{ml}$ \\
\hline
\end{tabular}

\section{Plasma}

Bei der Bestimmung des Plamaporphyrins bedienten wir uns der von SCHLENKER und Mitarbeitern (37) modifizierten Methode und arbeiteten nach Waldrons (38) Vorschriften. Das Verfahren beruht auf der Bindung des Porphyrins an Florisil ${ }^{1}$ ) und nach-

1) Florisil Harz ${ }^{60} / 100$ mesh $500 \mathrm{~F}$. folgender Eluierung; die Werte werden gleichfalls spektrophotometrisch festgehalten; infolge seiner Einfachheit, soivie des Vorteils, daß man nur eine relativ geringe Menge Plasma $(3-5 \mathrm{~m} /$ ) benötigt, hat sich das Verfahren in der Praxis gut bewährt. Die in pathologischer Menge vorhandenen Porphyrine lassen sich aus den Eluaten chromatographisch absondern, meist aber pflegt man nur den Gesamt-Porphyringehalt zu bestimmen.

Tab. 6

Normalwerte für Gesamtporphyrin im Plasma. Die eigenen Ergebnisse werden mit in der Literatur angegebenen verglichen

\begin{tabular}{|c|c|c|c|}
\hline Porphyrin & Autor & $\begin{array}{c}\text { Zitat } \\
\text { Nr. }\end{array}$ & gefundene Mengen \\
\hline \multirow[t]{2}{*}{ Gesamt- } & $\begin{array}{l}\text { PETERKA u. Mitarbeiter } \\
\text { SCHLENKER u. Mitarbeiter } \\
\text { WALDRON } \\
\text { WARD u. Mitarbeiter }\end{array}$ & $\begin{array}{l}15 \\
37 \\
38 \\
39\end{array}$ & $\begin{array}{l}0,0-0,1 \mu \mathrm{g} / 100 \mathrm{ml} \\
0,0-0,7 \mu \mathrm{g} / 100 \mathrm{ml} \\
0,0-0,9 \mu \mathrm{g} / 100 \mathrm{ml} \\
0,2-0,7 \mu \mathrm{g} / 100 \mathrm{ml}\end{array}$ \\
\hline & $\begin{array}{l}\text { GORECZKY, RóTH u. } \\
\text { BRECKNER }\end{array}$ & & $0,35 \pm 0,18 \mu \mathrm{g} / 100 \mathrm{ml}$ \\
\hline
\end{tabular}

\section{Galle}

Im Falle gewisser Krankheitsbilder, vor allem bei der hepatischen Porphyrie, wird der Porphyringehalt der Galle bestimmt. Natürlich eignet sich hierfür allein die „B-Galle“, welche nach intraduodenaler Einführung von Magnesiumsulfat gewonnen wird.

Die Bestimmung der einzelnen Porphyrine geschieht in der bei der Harnuntersuchung üblichen Weise; man extrahiert zunächst mit Äther, dann mit Äthylacetat und extrahiert danach die einzelnen Porphyrinarten aus den gewonnenen Extrakten mittcls Salzsäure. Die Normalwerte können bedeutend voneinander abweichen, vor allem, wenn die zur Untersuchung bestimmte Galle nicht nach Vorschrift gewonnenen wurde. Im allgemeinen wird hier das Koproporphyrin bestimmt.

Tab. 7

Normalwerte für Koproporphyrin in Galle. Die eigenen Ergebnisse werden mit in der Literatur angegebenen verglichen

\begin{tabular}{cccc}
\hline Porphyrin & Autor & Zitat Nr. & gefundene Menge \\
\hline Kopro- & $\begin{array}{c}\text { BRUGSCH } \\
\text { WATSON }\end{array}$ & 7 & $120 \mu \mathrm{g} / 100 \mathrm{ml}$ \\
\hdashline & $\begin{array}{c}\text { GORECZKY, RóTH u. } \\
\text { BRECKNER }\end{array}$ & 40 & $129 \mu \mathrm{g} / 100 \mathrm{ml}$ \\
\hline
\end{tabular}

Auf die Besprechung der Isolierung der Isomeren, die Veresterung und Kristallisation der Prophyrine wollen wir hier nicht eingehen, da dies nicht in das Gebiet der Diagnostik gehört und über die üblichen diagnostischen Verfahren hinausgeht. Zur Herstellung von gereinigten Standardpräparaten jedoch dürfte es nötig sein, diese Methoden anzuwenden. Sie fanden jedoch keine weitgehende Verbreitung in der Diagnostik. Eine einfachere Form der Differenzierung stellen die verschiedenen elektrophoretischen Methoden dar, über welche With (41), Ippen (42), Clotten und Wehinger (43) sowie Srmon und Kocsis (44) berichtet haben.

\section{Literatur}

1. Zieve, L., E. Hill, S. Schwartz und C. J. Watson, J. Laborat. Clin. Med. S. Louis 41, 663 (1959). - 2. Eales, L., S. Afr. J. Lab. clin. Med. 5, 63 (1960). - 3. Eales, L., E. B. Dowdle, S. J. SANDERS und G. D. Swéeney, S. Afr. Med. J. 37, 100 (1963). 4. Leading Article, Lancet 1967/I 663. - 5. HAEger-Aronsen, B., Scand. J. Clin. Laborat. Invest. 14, 397 (1962). - 6. HinsBerg, K. und K. Lang, Medizinische Chemie. Urban-Schwarzenberg,
München-Berlin (1951). - 7. Brugsch, J., Porphyrine, 2. Aufl. J. A. Barth, Leipzig (1959). - 8. FrenCH, J. M., M. T. ENGLAND, J. Lrines und E. Thonger, Arch. Biochem. Biophysics 107, 404 (1964). - 9. Käser, H., H. Koblet und G. Riva, Schweiz. med. Wschr. 93, 1052 (1963). - 10. Politzer, W. M. und I. Kessel, J. Clin. Path., London 11, 183 (1958). - 11. Mauzerall, D. und S. Granick, J. biol. Chemistry 219, 435 (1956). - 12. Gibson, 
K. D., W. G. Laver und A. Neuberger, Biochem. J. 70, 71 (1958). - 13. HeIlmeyer, L., Die Störungen der Bluthämsynthese mit besonderer Berücksichtigung der sideroachrestischen Anämien und erythropoetischen Porphyrien. 1. Aufl. G. Thieme Stuttgart (1964). - 14. Malooly, D. A. und N. C. Hrghtower, J. Laborat. Clin. Med. S. Louis 59, 568 (1962). - 15. Peterka, E. S., R. M. Fusara, W. J. Runge, M. O. Jaffe und C. J. Watson, J. Amer. Med. Ass. 193, 1036 (1965). - 16. Peters, H. A., S. Woods, P. L. ErchmanN und H. H. Reese, Ann. Int. Med. 47, 889 (1957). - 17. Prato, V., U. MazzA, A. L. Massaro und V. Battistini, Arch. Immunol. Ther. Exp. 15, 765 (1967). - 18. ReNNER, A., Aerztl. Wschr. 9, 201 (1958). - 19. Strch, W., Klin. Wschr. 37, 681 (1959). - 20. Róth, I. und L. GoreczKy, Zschr. inn. Med., Leipzig 16, 1078 (1961). - 21. Haeger-Aronsen, B., Scand. J. Clin. Laborat. Invest. 12. Suppl. (1960). - 22. Holtr, G., C. Rimington, B. C. TATE und G. Thomas, Quart. J. Med. 27, 1 (1958). - 23. RimingtON, C. und S. L. Svennsson, Scand. J. Clin. Laborat. Invest. 2, 209 (1950). - 24. Wrrt, T. K. Scand. J. Clin. Laborat. Invest. 7, 193 (1955). - 25. Rimington, C., Method Sheet No. 37, Publ. Unicam Instruments Ltd. Cambridge (1962). - 26. REINKINGH, W. A. und E. J. van Kampen, Clin. chim. Acta (Amsterdam) 9, 592 (1964). - 27. WALDENSTRöM, J. und B. HAEger-AronsEN,
Brit. Med. J. 1963/II 272. - 28. Watson, C. J., S. C. Rudolph und D. L. Аввот, J. Laborat. Clin. Med. S. Louis 41, 437 (1961). 29. Heilmeyer, L. und R. Clotren, Dtsch. med. Wschr. 87, 131 (1962). - 30. Schwartze, G., Dtsch. Gesd.wes. 22, 1660 (1967). 31. Barnes, H. D., S. Afr. med. J. 32, 680 (1958). - 32. Rimington, C., P. N. Morgan, K. Nrchols, J. D. Everalit und R. R. Davies, Lancet 1963/II 318. - 33. KaUtzSCHE, E. und J. F. Meyer, Med. Klin. 56, 1857 (1961). - 34. Donaldson, E. M., A. G. Donaldson und C. Rimington, Brit. Med. J. 1966/II 659. 35. LANGHof, H., L. HeiLmeYer, R. ClotTen und L. Rietschel, Dtsch. med. Wschr. 89, 1289 (1964). - 36. DAGG, J. H., A. GoldBERG und A. LochHeAd, Br. J. Haemat. 12, 326 (1966). - 37. Schienker, F. S., C. L. Davies und C. L. Krrchell, Amer. J. Clin. Path. 36, 31 (1961). - 38. WaldRON, H. A., Brit. J. Industr. Med. 21, 315 (1964). - 39. WARD, E. und H. L. Mason, J. Clin. Invest. 29, 905 (1950). - 40. Watson, C. J., R. Pimenta de Mello, S. Schwartz, V. E. Havkinson und I. Bossmaier J. Laborat. Clin. Med. S. Louis 37, 831 (1951). - 41. Wrrt, T. K., Scand. J. Clin. Laborat. Invest. 8, 113 (1956). - 42. IPpEN, H., Klin. Wschr. 40, 745 (1962). - 43. Clotren, R. und H. Wehrnger, Dtsch. med. Wschr. 87, 131 (1962). - 44. SṬmon, M. und G. Kocsis, Persönliche Mitteilung.
Doz. Dr. L. Goreczky MAV-Krankenhaus Budapest VI Rudas L. u. 111., Ungarn 